\title{
VLA cm-wave survey of young stellar objects in the Oph A cluster: constraining extreme UV- and X-ray-driven disk photoevaporation
}

\section{A pathfinder for Square Kilometre Array studies *}

\author{
A. Coutens ${ }^{1}$, H. B. Liu ${ }^{2}$, I. Jiménez-Serra ${ }^{3}$, T. L. Bourke ${ }^{4}$, J. Forbrich ${ }^{5}$, M. Hoare ${ }^{6}$, L. Loinard ${ }^{7,8}$, L. Testi ${ }^{2,9}$, \\ M. Audard ${ }^{10,11}$, P. Caselli1 ${ }^{12}$, A. Chacón-Tanarro ${ }^{13}$, C. Codella ${ }^{9,14}$, J. Di Francesco ${ }^{15,16}$, F. Fontani ${ }^{9}$, \\ M. Hogerheijde ${ }^{17,18}$, A. Johansen ${ }^{19}$, D. Johnstone ${ }^{15,16}$, S. Maddison ${ }^{20}$, O. Panić ${ }^{6}$, L. M. Pérez ${ }^{21}$, L. Podio ${ }^{9}$, \\ A. Punanova ${ }^{22}$, J. M. C. Rawlings ${ }^{23}$, D. Semenov ${ }^{24,25}$, M. Tazzari ${ }^{26}$, J. J. Tobin ${ }^{27}$, M. H. D. van der Wiel' ${ }^{28}$, \\ H. J. van Langevelde ${ }^{29,16}$, W. Vlemmings ${ }^{30}$, C. Walsh ${ }^{6}$, and D. Wilner ${ }^{31}$
}

(Affiliations can be found after the references)

Received 22 February 2019 / Accepted 4 September 2019

\begin{abstract}
Observations of young stellar objects (YSOs) in centimeter bands can probe the continuum emission from growing dust grains, ionized winds, and magnetospheric activity that are intimately connected to the evolution of protoplanetary disks and the formation of planets. We carried out sensitive continuum observations toward the Ophiuchus A star-forming region, using the Karl G. Jansky Very Large Array (VLA) at $10 \mathrm{GHz}$ over a field-of-view of $6^{\prime}$ and with a spatial resolution of $\theta_{\text {maj }} \times \theta_{\min } \sim 0 \prime^{\prime} 4 \times 00^{\prime} 2$. We achieved a $5 \mu \mathrm{Jy}^{\text {beam }}{ }^{-1}$ rms noise level at the center of our mosaic field of view. Among the 18 sources we detected, 16 were YSOs (three Class 0, five Class I, six Class II, and two Class III) and two were extragalactic candidates. We find that thermal dust emission generally contributed less than $30 \%$ of the emission at $10 \mathrm{GHz}$. The radio emission is dominated by other types of emission, such as gyro-synchrotron radiation from active magnetospheres, free-free emission from thermal jets, free-free emission from the outflowing photoevaporated disk material, and synchrotron emission from accelerated cosmic-rays in jet or protostellar surface shocks. These different types of emission could not be clearly disentangled. Our non-detections for Class II/III disks suggest that extreme UV-driven photoevaporation is insufficient to explain disk dispersal, assuming that the contribution of UV photoevaporating stellar winds to radio flux does not evolve over time. The sensitivity of our data cannot exclude photoevaporation due to the role of X-ray photons as an efficient mechanism for disk dispersal. Deeper surveys using the Square Kilometre Array (SKA) will have the capacity to provide significant constraints to disk photoevaporation.
\end{abstract}

Key words. stars: formation - protoplanetary disks - radio continuum: stars - stars: activity

\section{Introduction}

The first step towards forming the building blocks of planets occurs via grain growth in disks composed of dust and gas surrounding young stars (e.g., Testi et al. 2014; Johansen et al. 2014). Thus, the time available for the formation of planets is limited by the lifetime of the disk. After $10 \mathrm{Myr}$, the majority of disks disappear (e.g., Haisch et al. 2005; Russell et al. 2006; Williams \& Cieza 2011; Ribas et al. 2015). Understanding the mechanisms that lead to disk dispersal and the time-scales involved is crucial in characterizing the environment in which planets are formed.

The detection of transition disks where dust has been cleared within the inner regions (e.g., Strom et al. 1989; Pascucci et al. 2016; van der Marel et al. 2018; Ansdell et al. 2018) has favored the development of theoretical models where disk dispersal occurs from the inside out (e.g., photoevaporation, grain growth, giant planet formation). In particular, models of disk dispersal through photoevaporation can successfully explain inner hole sizes and accretion rates for a large number of transition disks

\footnotetext{
* The mosaic image is only available at the CDS via anonymous ftp to cdsarc.u-strasbg.fr $(130.79 .128 .5)$ or via http:// cdsarc.u-strasbg.fr/viz-bin/cat/J/A+A/631/A58
}

(e.g., Alexander \& Armitage 2009; Owen et al. 2011, 2012; Ercolano et al. 2018). Given that radio observations trace ionized material, they could therefore provide useful constraints on different photoevaporation models (Pascucci et al. 2012; Macías et al. 2016). Moreover, radio observations are also useful for tracing the magnetospheric activity of young stellar objects (YSOs), as well as grain growth process in disks (Güdel 2002; Forbrich et al. 2007, 2017; Choi et al. 2009; Guilloteau et al. 2011; Pérez et al. 2012; Liu et al. 2014; Tazzari et al. 2016).

The Ophiuchus A (Oph A) cluster is one of the nearest star-forming regions $(d \sim 137 \mathrm{pc}$, Ortiz-León et al. 2017). Its proximity and the abundance of YSOs at a wide range of evolutionary stages (Gutermuth et al. 2009) make this cluster an ideal laboratory for studying the evolution of YSO radio activity. We present here the first results of new radio continuum observations of the Oph A region using the NRAO Karl G. Jansky Very Large Array (VLA) at $10 \mathrm{GHz}$, which have achieved an unprecedented level of sensitivity ( $5 \mu \mathrm{Jy}$ beam $^{-1}$ in the center of the field). In Sect. 2, we describe the observations and data reduction. In Sect. 3, we present the sources that have been detected and we analyze the nature of the continuum emission detected towards the YSOs. In Sect. 4, we discuss the contribution of the extreme ultraviolet (EUV) and X-ray photoevaporation in the dispersal of 


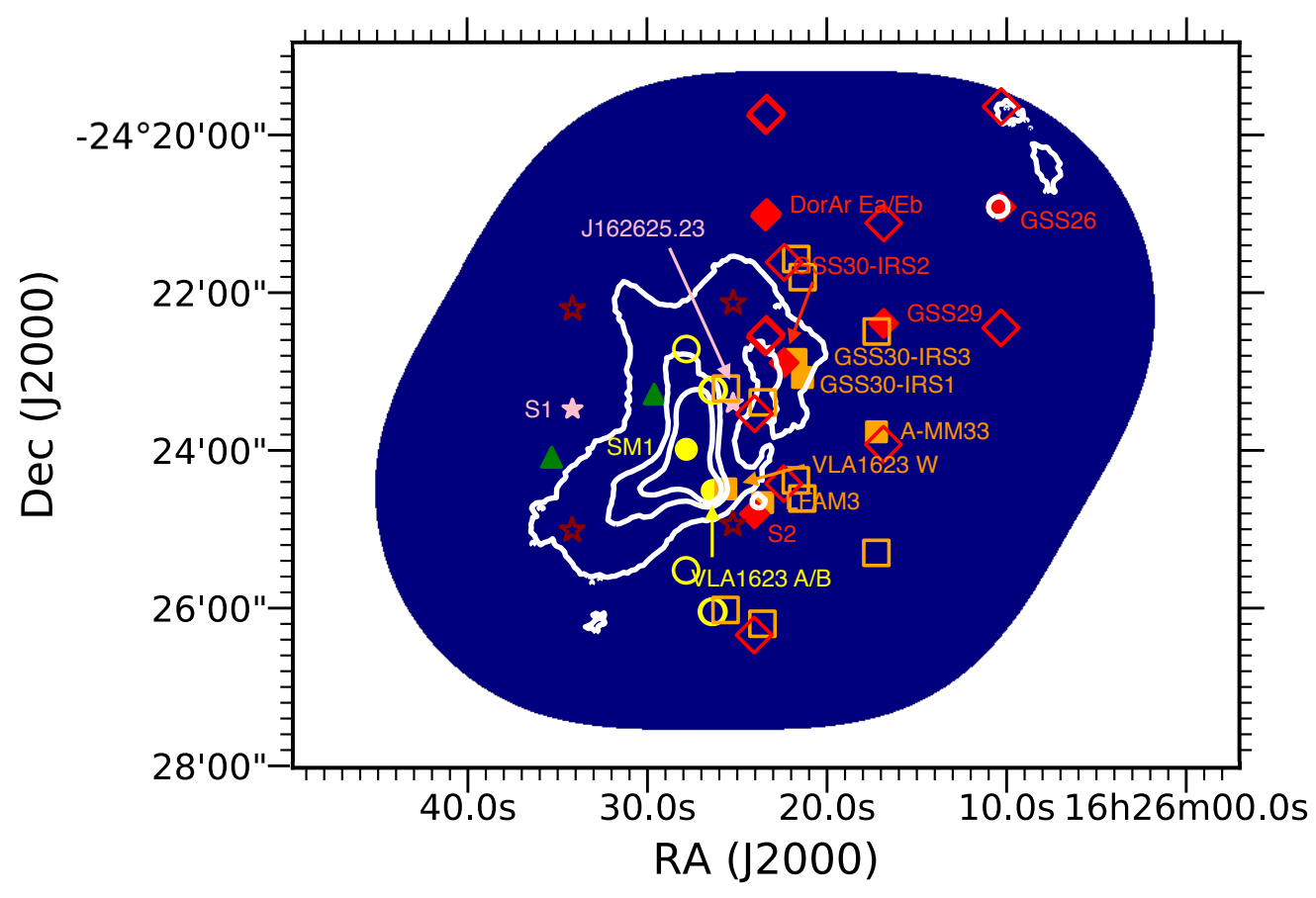

Fig. 1. Field of view covered by VLA $X$ band observations shown in blue. The position of the detected Class 0, I, II and III sources are indicated with yellow circles, orange squares, red diamonds, and pink stars, respectively. Sources VLA1623 and DoAr 24E are binary systems. The extragalactic candidates are indicated with green triangles. White contours represent $850 \mu \mathrm{m}$ continuum observations from the JCMT Gould Belt Survey taken by SCUBA-2 (Pattle et al. 2015; Kirk et al. 2018). disks in Oph A, and the prospects related to the upcoming Square Kilometre Array (SKA).

\section{Observations}

We performed five epochs of mosaic observations towards the Oph A YSO cluster at $X$ band $(8.0-12.0 \mathrm{GHz})$ using the VLA (project code: 16B-259, PI: Audrey Coutens). All five epochs of observation (see Table 1) were carried out in the most extended, A array configuration, which provides a projected baseline range from 310 to $34300 \mathrm{~m}$. We used the 3-bit samplers and configured the correlator to have $4 \mathrm{GHz}$ of continuous bandwidth coverage centered on the sky frequency of $10 \mathrm{GHz}$ divided into 32 contiguous spectral windows. The pointing centers of our observations are given in Table 2. They are separated by $2.6^{\prime}$, while the primary beam FWHM is $4.2^{\prime}$. In each epoch of observation, the total on-source observing time for each pointing was $312 \mathrm{~s}$. The quasar J1625-2527 was observed approximately every $275 \mathrm{~s}$ for complex gain calibration. We observed $3 \mathrm{C} 286$ as the absolute flux reference. The joint imaging of these mosaic fields forms an approximately parallelogram-shaped, mosaic field of view, of which the width and height are $\sim 6^{\prime}$. Figure 1 shows the observed field of view.

We calibrated the data manually using the $\mathrm{CASA}^{1}$ software package, following standard data calibration procedures. To maximize sensitivity, we combined the data from all five epochs of observation. We ensured that highly variable sources did not affect the image quality or the results by additionally imaging the individual epochs separately (see Sect. 3.2.2). The imaging was done with Briggs robust $=2.0$ weighting, gridder $=$ "mosaic", specmode $=$ "mfs", and nterms $=1$. This setting was used to maximize signal-to-noise ratio $(\mathrm{S} / \mathrm{N})$ ratios. Using $>1$ nterms is not suitable for this project given the sources are relatively faint. At the average observing frequency, we obtained a synthesized beam of $\theta_{\text {maj }} \times \theta_{\text {min }} \sim 0^{\prime} .4 \times 0^{\prime}$. 2 and a maximum detectable angular scale of $\sim 5^{\prime \prime}$ (or $\sim 700 \mathrm{au}$ ). After primary

\footnotetext{
1 The Common Astronomy Software Applications software package, release 4.7.2 (McMullin et al. 2007).
}

Table 1. VLA observations.

\begin{tabular}{lclll}
\hline \hline Epoch & $\begin{array}{c}\text { Starting time } \\
(\mathrm{UTC})\end{array}$ & $\begin{array}{l}\text { Initial API rms } \\
\left({ }^{\circ}\right)\end{array}$ & $\begin{array}{l}\text { Projected baseline } \\
(\mathrm{m})\end{array}$ & $\begin{array}{c}F_{9.9 \mathrm{GHz}}^{\text {gain }} \\
(\mathrm{Jy})\end{array}$ \\
\hline 1 & $2016-12-0221: 31$ & 11 & $310-34300$ & 1.4 \\
2 & $2016-12-0521: 18$ & 6.0 & $460-34300$ & 1.3 \\
3 & $2017-01-0618: 05$ & 13 & $325-32800$ & 1.4 \\
4 & $2017-01-1418: 40$ & 13 & $310-34300$ & 1.3 \\
5 & $2017-01-2217: 12$ & 4.4 & $665-33100$ & 1.3 \\
\hline
\end{tabular}

Notes. API refers to Atmospheric Phase Interferometer, which observes an $11.7 \mathrm{GHz}$ beacon from a geostationary satellite with a 300 meters baseline. $F_{9.9 \mathrm{GHz}}^{\text {gain }}$ is the measured flux of the gain calibrator J16252527.

Table 2. Mosaic pointings.

\begin{tabular}{lll}
\hline \hline Name & $\begin{array}{l}\text { RA } \\
(\mathrm{J} 2000)\end{array}$ & $\begin{array}{l}\text { Dec } \\
(\mathrm{J} 2000)\end{array}$ \\
\hline X1 & $16^{\mathrm{h}} 26^{\mathrm{m}} 32^{\mathrm{s}} .00$ & $-24^{\circ} 24^{\prime} 30^{\prime} .^{\prime} 0$ \\
X2 & $16^{\mathrm{h}} 26^{\mathrm{m}} 20^{\mathrm{s}} .62$ & $-24^{\circ} 24^{\prime} 30^{\prime} 0$ \\
X3 & $16^{\mathrm{h}} 26^{\mathrm{m}} 26^{\mathrm{s}} .31$ & $-24^{\circ} 22^{\prime} 15^{\prime \prime} 0$ \\
X4 & $16^{\mathrm{h}} 26^{\mathrm{m}} 14^{\mathrm{s}} .93$ & $-24^{\circ} 22^{\prime} 15^{\prime \prime} .0$ \\
\hline
\end{tabular}

beam correction, we achieved a rms noise level of $\sim 5 \mu \mathrm{Jy}_{\text {beam }}^{-1}$ at the center of our mosaic field, degraded to $\sim 28 \mu \mathrm{Jy} \mathrm{beam}^{-1}$ toward the edges of the mosaic. The flux calibration uncertainty is expected to be about $5 \%$.

\section{Results}

\subsection{Source census and comparison with other surveys}

In total, we detected 18 sources above $5 \sigma$ in our mosaic field of view. The fluxes of the detected sources were measured by performing two-dimensional Gaussian fits, using the 
imfit task of CASA. The derived fluxes and coordinates can be found in Table B.1, where the names of the sources Jhhmmss.ss-ddmmss.s are based on the coordinates of peak intensity obtained with the fitting procedure. The position uncertainties are typically about a few tens of mas. Table B.1 also lists the more commonly used names of these sources. When the source structure was too complex to be fitted with this method or the results of the fit were too uncertain, we measured the flux by integrating over a circular area around the source with CASA. Table A.1 summarizes the sizes measured with the Gaussian fit after deconvolution from the beam.

We compared our detections with the list of YSOs present in our field based on the photometric and spectroscopic surveys presented in Wilking et al. (2008), Jørgensen et al. (2008), Hsieh \& Lai (2013), and Dzib et al. (2013). Dzib et al. (2013) carried out large-scale observations of the Ophiuchus region with the VLA at 4.5 and $7.5 \mathrm{GHz}$ with a resolution of $1^{\prime \prime}$. Wilking et al. (2008) used X-ray and infrared photometric surveys as well as spectroscopic surveys of the L1688 cloud to list all the association members present in the Two Micron All-Sky Survey (2MASS) catalog. They also classify sources according to their respective spectral energy distributions (SEDs) built from the Spitzer Cores to Disks (c2d) survey. Hsieh \& Lai (2013) compile another list based on the $\mathrm{c} 2 \mathrm{~d}$ Legacy Project after developing a new method to identify fainter YSOs based on analyzing multidimensional magnitude space. Finally, Jørgensen et al. (2008) identify the more deeply embedded YSOs by jointly analyzing Spitzer and JCMT/SCUBA data. Overall, 18 of our detected sources have all been found in at least one previous catalog or study. Specifically, 16 of our 18 radio detections are associated with YSOs, while the remaining two are probably extragalactic sources (Dzib et al. 2013). Individual images of our detected YSOs are provided in Fig. 2. Sidelobes are visible for some of these sources (S1, SM1). In total, we detected 11 YSO candidates listed in the catalog of Wilking et al. (2008), while the remaining 19 YSOs in that catalog were undetected (see label "b" in Table B.1). Also, we detected nine of the sources listed by Hsieh \& Lai (2013, see label "c" in Table B.1). Finally, we detected five of the young sources listed in Jørgensen et al. (2008), while two others (162614.63-242 507.5 and 162 625.49$242301.6)$ were undetected.

Compared to the previous VLA survey at 4.5 and $7.5 \mathrm{GHz}$ by Dzib et al. (2013, see Table B.1), we detected seven additional radio sources, namely J162627.83-242 359.4 (SM1, \#3 in Table B.1), J162617.23-242345.7 (A-MM33, \#4), J162621.36-242 304.7 (GSS30-IRS1, \#5), J162623.36-242 059.9 (DoAr 24Ea, \#19), J162623.42-242 102.0 (DoAr 24Eb, \#20), J162624.04-242 448.5 (S2, \#21), and J162625.23-242324.3 (\#30). All of these are young stellar objects. Three sources reported in Dzib et al. (2013) were undetected in our observations. These three sources are extragalactic (EG) candidates. A possible explanation for these non-detections is that they have a negative spectral index. Hence, the observations at 4.5 and $7.5 \mathrm{GHz}$ by Dzib et al. (2013) could be more sensitive to this type of target because of their greater brightness at a lower frequency. Another explanation would be that these sources are variable. We comment briefly on some of the individual young stellar objects below.

J162627.83-242 359.3 (also known as SM1, \#3) was previously classified as a prestellar core (see Motte et al. 1998). It was, however, detected at $5 \mathrm{GHz}$ with the VLA at an angular resolution of $\sim 10^{\prime \prime}$ (measured peak fluxes of 130-200 $\mu \mathrm{Jy}$ beam $^{-1}$; Leous et al. 1991; Gagné et al. 2004), although, in the first study, the source appears slightly offset by $3^{\prime \prime}$. More recent ALMA observations suggest that SM1 is actually protostellar and that it hosts a warm $(\sim 30-50 \mathrm{~K})$ accretion disk or pseudo-disk (Friesen et al. 2014, 2018; Kirk et al. 2017).

The source J162623.42-242 101.9 (known as DoAr 24Eb, \#20) is the companion of the protostar J162623.36-242059.9 (DoAr 24Ea, \#19), also detected in our dataset (see Fig. 2). These two sources are assumed to be at a similar evolutionary stage, although more data are needed to confirm this hypothesis (Kruger et al. 2012).

It has been suggested that the source J162634.17-242 328.7 (S1, \#32) is a binary separated by 20-30 mas (see discussion in Ortiz-León et al. 2017). Our VLA $X$ band image does not spatially resolve the individual binary components. We note that the secondary component was not detected in the most recent epochs covered by Ortiz-León et al. (2017).

For the sources we did not detect, we evaluated the $3 \sigma$ upper limits, which varied across the mosaic field due to primary beam attenuation (see Table B.1). For $3 \sigma$ rms levels as low as $\sim 15 \mu \mathrm{Jy}_{\text {beam }^{-1}}$, the detection statistics at $10 \mathrm{GHz}$ in this region are $3 / 3$ for Class 0 sources (100\%), 5/8 for Class I YSOs (63\%), $6 / 16$ Class II sources (38\%), and 2/5 Class III objects (40\%).

Figure 3 shows the radio emission properties of the YSOs versus their Spitzer [3.6]-[4.5] colors (Evans et al. 2009). We see that the measured fluxes at $10 \mathrm{GHz}$ of some sources are significantly brighter than the fluxes measured at $7.5 \mathrm{GHz}$ by Dzib et al. (2013), while for other sources it is the opposite. The absence of a systematic trend indicates that our data are not likely to have been affected by flux calibration issues. We note that the classification of the continuous evolution of YSOs into Class 0/I, II, and III stages, taken from the literature, is to some extent artificial, and can be uncertain for YSOs that are transitioning from one stage to another. In addition, different catalogs or databases may report slightly different classifications, which are noted in Table B.1.

\subsection{Nature of the emission at $10 \mathrm{GHz}$}

In this section, we evaluate how much of the flux measured towards the YSOs in our $10 \mathrm{GHz}$ VLA observations is due to: (i) thermal emission from dust, and (ii) other mechanisms such as free-free emission from ionized radio jets or photoevaporative winds, gyro-synchrotron emission from active magnetospheres, and synchrotron emission produced through the acceleration of cosmic-rays by jet or protostellar surface shocks (e.g., Macías et al. 2016; Gibb 1999; Forbrich et al. 2007; Padovani et al. 2016; Padovani \& Galli 2018).

The brightest source in our sample, J162634.17-242 328.7 (S1, \#32), has been already investigated in several studies and is known to be a completely non-thermal source. There is no evidence of a free-free component (e.g., André et al. 1988; Loinard et al. 2008; Ortiz-León et al. 2017). Indeed, the flux measured with the Very Long Baseline Array (VLBA) is systematically found to be equal to the VLA flux (Loinard et al. 2008; Ortiz-León et al. 2017). Since the VLBA is only sensitive to non-thermal emission, whereas the VLA is, in principle, sensitive to both thermal and non-thermal emission (e.g., Ortiz-León et al. 2017), the emission of this source is confirmed here as fully non-thermal. This source is, however, quite peculiar, since the non-thermal emission is not strongly variable, as has been confirmed in our observations (see Table B.1). This result is somewhat of a mystery, and may be due to a magnetic field that, in this specific case, is fossil-based rather than dynamodriven (André et al. 1988). The former would explain S1's lack of flaring activity that would typically be seen in non-thermal 

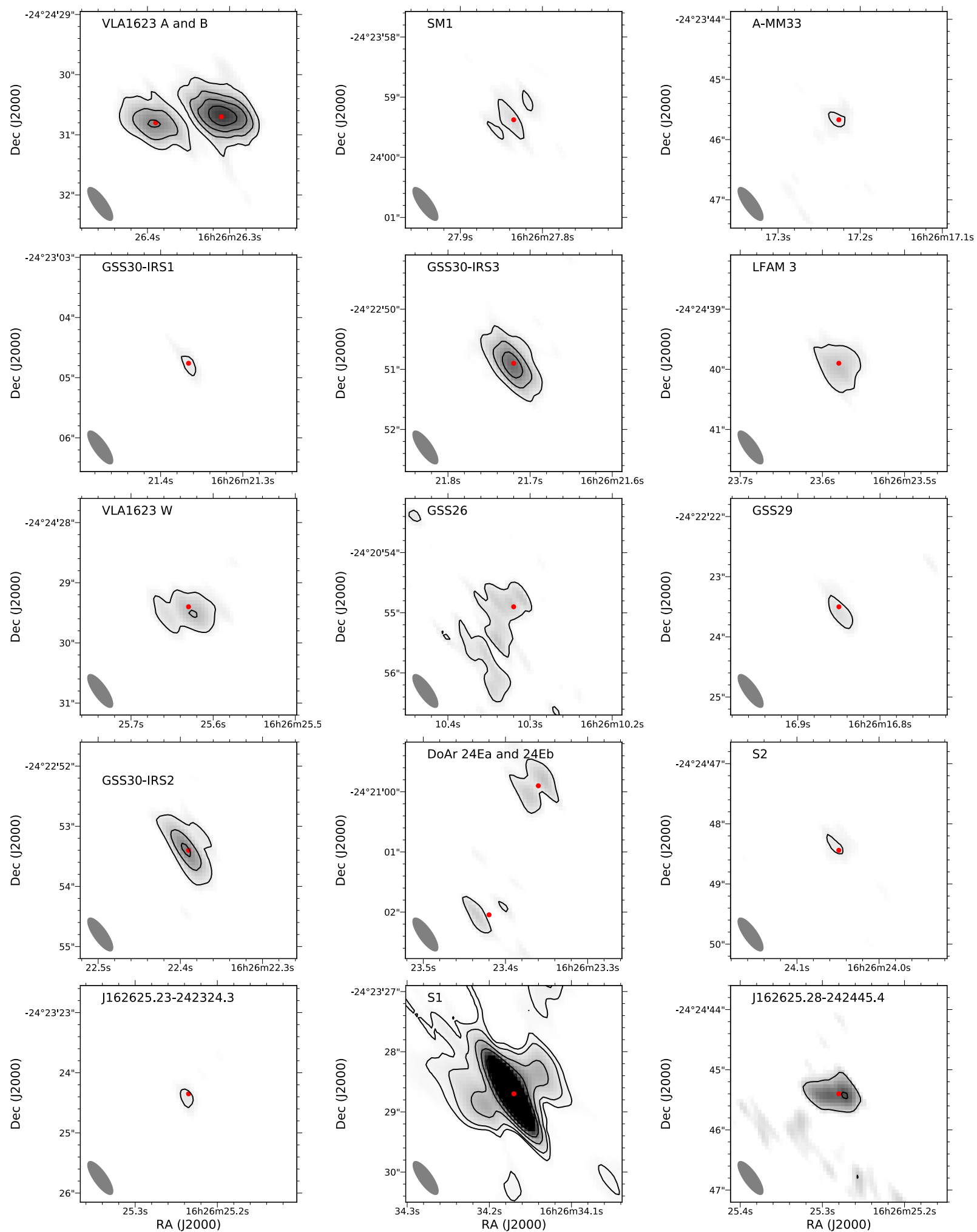

Fig. 2. Continuum observations of young stellar objects detected with VLA in band $X$. For all sources except S1, contours start from $5 \sigma$ with a step of $5 \sigma$. For S1, contours are 10,20,50,100, and 200 $\sigma$. Greyscale images start at $3 \sigma$. The red dot corresponds to the coordinates used to name the sources in Table B.1. Last map (J162625.28-242 445.4) is for epoch 3 only.

sources otherwise. Given these extended studies focused on the S1 source, we do not discuss this here and further.

\subsubsection{Contribution of thermal emission from dust}

To determine the thermal contribution from dust, we assume that the $\sim 107 \mathrm{GHz}$ continuum fluxes reported by Kirk et al. (2017) are entirely due to dust thermal emission, and then extrapolate the contribution of dust emission at our observing frequency of $10 \mathrm{GHz}$ by assuming a power-law with a spectral index $\alpha$ (see Table B.1). We note that the angular resolution of the observations reported by Kirk et al. (2017) is approximately 10 times coarser than that of our VLA observations. Therefore, 


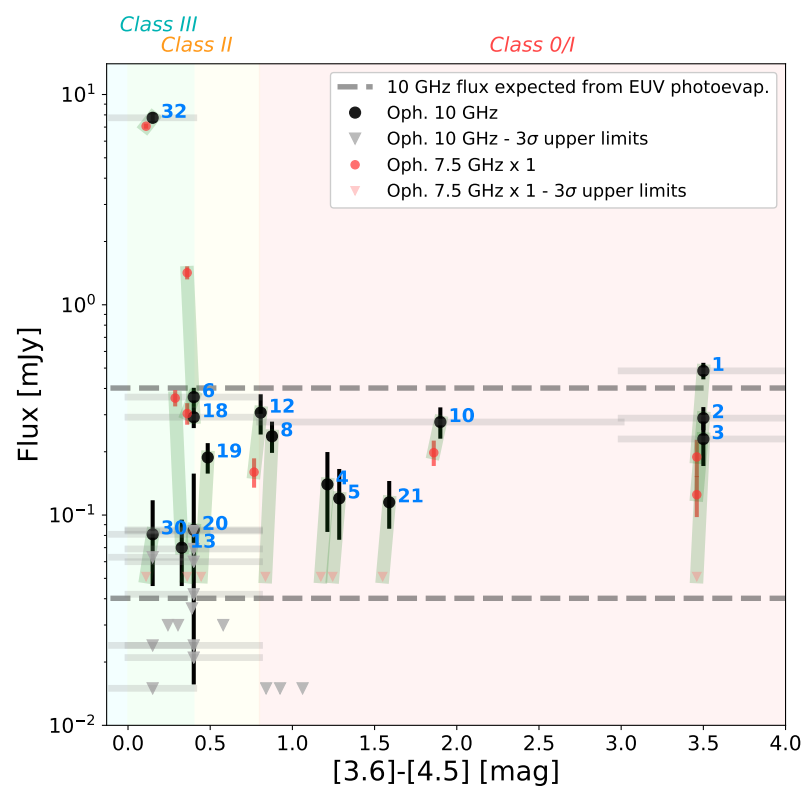

Fig. 3. Summary of observed $10 \mathrm{GHz}$ radio fluxes. We show the characteristic [3.6]-[4.5] color ranges of the Class III, II, and 0/I YSOs as blue, yellow, and red filled regions which are bound in horizontal axis by $[-0.4,0.4],[0.0,0.8]$, and $[0.8,4.0]$, respectively (overlapped area for Class III and II objects appears in green; see Allen et al. 2004). Our $10 \mathrm{GHz}$ detections are presented as black circles. For sources we detected at $10 \mathrm{GHz}$, we also presented the fluxes measured at $7.5 \mathrm{GHz}$ (red symbols) by Dzib et al. (2013). For the purposes of presentation, we offset the [3.6]-[4.5] values of the red symbols by -0.04 . The observations towards the same target sources are linked by green lines. Gray and red downward triangles are the $3 \sigma$ upper limits from these observations. Dashed lines show the expected radio fluxes from EUV photoevaporation winds from protoplanetary disks, assuming the EUV flux $\Phi_{\mathrm{EUV}}=10^{41}$ (bottom) and $10^{42}$ photons s$^{-1}$ (top) .

our estimates of $10 \mathrm{GHz}$ dust emission should be regarded as the upper limits.

In the millimeter bands (e.g., $\sim 90-350 \mathrm{GHz}$ ), the spectral indices of Class 0/I objects may be $\alpha=2.5-3$ (see Chiang et al. 2012; Tobin et al. 2013, 2015; Miotello et al. 2014), while those of Class II/III objects may be lower ( $\alpha=2-2.5$; Ricci et al. 2010; Pérez et al. 2012; Tazzari et al. 2016) due to dust grain growth or high optical depths (see Li et al. 2017; Galván-Madrid et al. 2018). Taking this difference into account, we find that dust thermal emission could account for up to $\sim 30 \%$ of the observed $10 \mathrm{GHz}$ flux toward the Class 0 YSOs and is almost negligible in the Class III objects of our sample. For the Class I/II YSOs, the situation is more complex. In general, the contribution of the dust emission is $\leq 30 \%$ and in some cases, it is negligible. Exceptions, however, include the Class II sources J162610.32-242054.9 (also known as GSS26, \#12), for which dust emission could account for $\sim 80 \%$ of the continuum flux at $10 \mathrm{GHz}$, and 162 618.98-242414.3 (also called CRBR15, \#16), for which the predicted dust emission is higher than the upper limit of $15 \mu \mathrm{Jy} \mathrm{beam}^{-1}$, as well as two Class I sources (162625.49242 301.6, \#9 and 162 630.47-242 257.1, \#11), for which the predicted dust emission fluxes are comparable to the measured upper limits of $15 \mu \mathrm{Jy}$ beam $^{-1}$ at $10 \mathrm{GHz}$. Therefore, except for a few Class I/II sources, the contribution from dust is in general $\leq 30 \%$ of the total emission. This behavior is consistent with even higher-angular resolution $870 \mu \mathrm{m}$ ALMA observations toward the Class II sources J162623.36-242 059.9 (\#19) and J162623.42-242 101.9 (\#20, Cox et al. 2017), for which the dust contribution at $10 \mathrm{GHz}$ is also estimated to be $\leq 30 \%$ assuming dust spectral indices $\alpha=2-2.5$.

\subsubsection{Nature of the remaining radio emission}

The remaining radio fluxes are likely to have contributions from (thermal) free-free emission from ionized radio jets, (thermal) free-free emission due to photoevaporative winds (e.g., Macías et al. 2016) or (non-thermal) gyro-synchrotron emission from stellar magnetospheres (e.g., Gibb 1999; Forbrich et al. 2007). Jet or protostellar surface shocks can also produce (non-thermal) synchrotron emission at our observing frequency, for example, through the acceleration of cosmic-rays (Carrasco-González et al. 2010; Padovani et al. 2016; Anglada et al. 2018). These radio emission mechanisms present specific characteristics, which we describe below.

Free-free emission from thermal jets and (gyro-)synchrotron emission are known to be time-variable (Forbrich et al. 2007; Dzib et al. 2013), but they may have very different characteristic timescales (Liu et al. 2014). Free-free emission may vary on time-scales from a few weeks to a few months considering the ionized gas recombination timescales as well as the dynamical timescales of the inner $\sim 1$ au disk. Gyro-synchrotron emission, however, is expected to vary on shorter timescales (minutes) due to flares on a stellar surface, and can vary up to the rotational periods of protostars. These periods can be as long as $\sim 10$ days, due to large magnetic loops coupling protostars and their inner disks (Forbrich et al. 2007; Liu et al. 2014). Synchrotron emission is also expected to be variable, although the timescale is unclear (Padovani et al. 2016).

Observations also indicate that the fluxes of thermal (freefree) sources rarely vary more than $20-30 \%$, while, in general, non-thermal sources show greater variability (Ortiz-León et al. 2017; Tychoniec et al. 2018). The spectral indices of each type of emission can also differ. Free-free emission is characterized by spectral indices in the range $[-0.1,2.0]$, while gyro-synchrotron emission can span a significantly larger range of -5 to +2.5 . Spectral indices $<-0.4$ have been observed in YSO jets and attributed to synchrotron emission (Anglada et al. 2018).

To probe the origins of the detected emission, we first checked if any of our sources had also been detected with the VLBA. As explained at the beginning of Sect. 3.2, any detection with the VLBA is necessarily non-thermal. In addition to S1, three other sources present in our observations: J162616.85242223.5 (GSS 29, \#13), J162622.39-242 253.4 (GSS 30-IRS2, \#18), and J162625.63-242 429.4 (VLA1623 W, \#10) are detected at $5 \mathrm{GHz}$ with the VLBA (Ortiz-León et al. 2017) but they are undetected at $8 \mathrm{GHz}$ (see Table 3). By comparing the VLBA fluxes to the VLA fluxes measured by Dzib et al. (2013), we find that the emission of J162616.85-242 223.5 (\#13) could be fully non-thermal at $5 \mathrm{GHz}$. Unfortunately, no flux is available at $8 \mathrm{GHz}$ for this source and we cannot rule out a fully non-thermal emission at $10 \mathrm{GHz}$. The emission of J162622.39-242 253.4 (\#18) could be just partially non-thermal, as the VLBA flux is lower than the VLA flux (19\% at $5 \mathrm{GHz}$ and $<6 \%$ at $8 \mathrm{GHz}$ ). Nevertheless, this ought to be considered carefully as this source may possibly be highly variable (Dzib et al. 2013) and because the observations were not carried out in a similar timeframe. The emission of J162625.63-242 429.4 (\#10) could be fully nonthermal since at $5 \mathrm{GHz}$ the VLBA emission is higher than the VLA flux, and the VLBA upper limit at $8 \mathrm{GHz}$ is not lower than the VLA measurement even by a factor of 2 .

Next, we determined the spectral indices of all sources between $10 \mathrm{GHz}$ and $7.5 \mathrm{GHz}$ and between $10 \mathrm{GHz}$ and 
Table 3. Comparison of fluxes (mJy) measured towards 3 YSOs with VLBA and VLA.

\begin{tabular}{cccccc}
\hline \hline$\#$ & \multirow{2}{*}{ Source } & $\mathrm{VLBA}^{(1)}$ & $\mathrm{VLA}^{(2)}$ & $\mathrm{VLBA}^{(1)}$ & $\mathrm{VLA}^{(2)}$ \\
& & $5 \mathrm{GHz}$ & $5 \mathrm{GHz}$ & $8 \mathrm{GHz}$ & $8 \mathrm{GHz}$ \\
\hline 10 & $\mathrm{~J}_{162625.63-242} 429.4$ & 0.66 & 0.22 & $<0.12$ & 0.20 \\
13 & $\mathrm{~J} 162616.85-242223.5$ & $0.15-0.47^{(a)}$ & 0.34 & $\ldots$ & 0.36 \\
18 & $\mathrm{~J}_{162622.39-242253.4}$ & $0.30-0.38^{(a)}$ & 2.02 & $<0.09$ & 1.42 \\
\hline
\end{tabular}

Notes. ${ }^{(a)}$ The flux of these sources is known to vary.

References. ${ }^{(1)}$ From Ortiz-León et al. (2017). ${ }^{(2)}$ From Dzib et al. (2013).

4.5 GHz, taking into account both the fit uncertainty and the calibration uncertainty (see Col. 3 in Table 4). The only two sources with negative spectral indices (J162616.85-242 223.5, $\# 13$ and J162622.39-242 253.4, \#18) are those detected with the VLBA, which confirms the non-thermal origin of these sources' emission. Four sources, J162626.31-242 430.7 (\#1), J162627.83242359.4 (\#3), J162623.58-242439.9 (\#8), and J162623.36242059.9 (\#19), show spectral indices higher than 2.5, which may indicate variability (see below).

Finally, we explored the long-term variability of the YSOs. Our observations were averaged over a couple of months and compared with those of Dzib et al. (2013) obtained in 2011. Dzib et al. (2013) reports that seven out of the 16 YSOs we detected are variable (see Table B.1, Col. 9). We note that among these variable sources, two have spectral indices between 7.5 and $10 \mathrm{GHz}$ higher than 2.5. They also include the sources of non-thermal emission detected with the VLBA.

Any short-term variability will be explored in another paper by analyzing the 5 epochs separately, as well as more recent observations at lower spatial resolution. Nevertheless, to ensure that our conclusions are not affected by significant variability, we checked the maps of the different epochs individually. As expected, the faintest sources are barely detected or not detected at all depending on the noise level of each epoch. Among the brightest sources, even if some variations are observed for some, the fluxes vary around the values measured in the map with the combined epochs. We did not observe any cases where the flux is significantly higher at one epoch. The only exception is the source J162625.8-242445.0 (\#22), which is not detected in the map with the combined epochs, but clearly detected in epoch 3 with a flux of $0.4 \mathrm{mJy}$ (see Fig. 2), which is probably due to a non-thermal flare.

To explore possible radio flux variations since the observations of Dzib et al. (2013), we extrapolate their fluxes at $7.5 \mathrm{GHz}$ to those at $10 \mathrm{GHz}$, assuming that $\alpha$ is in the range of $[-0.1,2.0]$ (i.e., free-free emission from optically thin to optically thick limits) and compare the resulting values to our measured fluxes. For sources which were not detected at $7.5 \mathrm{GHz}$ by Dzib et al. (2013), we evaluate the corresponding $3 \sigma$ limits at $10 \mathrm{GHz}$ assuming $\alpha=2.0$, and compare the resulting values with our measurements (see Fig. 4). We find that there are three sources (J162626.31-242 430.7/\#1, J162616.85242 223.5/\#13, and J162622.39-242 253.4/\#18) detected in both our $10 \mathrm{GHz}$ observations and the previous $7.5 \mathrm{GHz}$ observations, for which the flux differences are too large to be explained by constant free-free emission. The emission of J162616.85242223.5 (\#13), and J162622.39-242 253.4 (\#18) is certainly non-thermal, as explained before. The emission of J162626.31242430.7 (\#1) may be explained either by non-thermal radio emission or by thermal radio flux variability of more than several tens of percent (see Fig. 4). In addition, after considering the spectral index range $[-0.1,2.0]$, it appears that three of our new radio detections (J162627.83-242 359.4/\#3, J162623.58-242 439.9/\#8, and J162623.36-242 059.9/\#19) cannot be attributed to our improved sensitivity. The measured $10 \mathrm{GHz}$ fluxes in the new VLA observations are significantly greater than $10 \mathrm{GHz}$ fluxes scaled from the $7.5 \mathrm{GHz}$ upper limit fluxes of Dzib et al. (2013) (see Fig. 4). Therefore, these detections were either due to variability or non-thermal, gyrosynchrotron spectral indices. The fractional radio flux variability of the sources can be seen in Fig. 4. We find that six out of our detected sources in the [3.6]-[4.5] color range of [0, 2] (i.e., late Class 0/I to early Class III stages) demonstrate over $50 \%$ fractional radio flux variability. The absolute values of their flux variations appear comparable to the observed flux variations from five epochs of observations towards CrA on the same date (Liu et al. 2014). The radio emission of some of these six sources (including J162625.63-242 429.4/\#10, 162 616.85242 223.5/\#13, and J162622.39-242 253.4/\#18) may be largely contributed by gyro-synchrotron emission, which can vary on short timescales.

Table 4 summarizes our conclusions regarding radio emission of the Oph A YSOs.

\subsubsection{Association with $\mathrm{X}$-ray emission}

We checked the sources associated with X-ray emission (Imanishi et al. 2003, see Col. 10 in Table B.1). For Class III sources, X-ray emission mainly arises from magnetized stellar coronae, while in younger (Class I/II) sources, additional mechanisms can produce X-ray emission (e.g., shocks due to the material infalling from the disk to the stellar surface or due to the interaction of outflows with circumstellar material). All the Class II and III sources detected in our data are associated with X-ray emission, apart from J162623.42-242 102.0 (DoAr 24Eb, \#20). The spatial resolution of the Chandra telescope might not be sufficient to separate its emission from J162623.36-242 059.9 (DoAr 24Ea). Among the younger sources we detected, only the Class I object J162623.58-242 439.9 (\#8) was detected in X-ray.

\section{Discussion: revisiting photoevaporation in Class II/III proto-planetary disks}

High-energy stellar photons (UV or X-rays) may contribute to the dispersal of protoplanetary disks through photoevaporation (Hollenbach et al. 1994; Alexander et al. 2014). The exact contribution of this mechanism to disk dispersal and the way it impacts planet formation, however, need to be investigated further. Observations at radio wavelengths can probe the freefree emission from a disk surface that is partially or totally ionized by EUV photons or X-ray photons. Therefore, radio wavelength observations can serve as a powerful diagnostic of the contributions of these two types of photons in protoplanetary disk evolution. For example, Pascucci et al. (2012) predict the level of radio emission expected from photoevaporation driven by EUV photons or X-ray photons. Based on an analysis of 14 circumstellar disks, Pascucci et al. (2014) then determines that the EUV photoevaporation mechanism may not play a significant role in disk mass dispersal, when EUV photon luminosities $\left(\Phi_{\text {EUV }}\right)$ are lower than $10^{42}$ photons s ${ }^{-1}$. Similar conclusions are obtained by Galván-Madrid et al. (2014) for ten disks toward the Corona Australis $(\mathrm{CrA})$ star-forming region, inferring $\Phi_{\mathrm{EUV}}<$ $(1-4) \times 10^{41}$ photons $\mathrm{s}^{-1}$, and by Macías et al. (2016) for the transitional disk of GM Aur $\left(\Phi_{\mathrm{EUV}} \sim 6 \times 10^{40}\right.$ photons s $\left.^{-1}\right)$. 
Table 4. Summary of the emission of the YSOs.

\begin{tabular}{|c|c|c|c|c|c|c|c|c|}
\hline \multirow[t]{2}{*}{ \# } & \multirow[t]{2}{*}{ Source } & \multirow{2}{*}{$\begin{array}{c}\text { Spectral } \\
\text { index } \\
\alpha^{(1)}\end{array}$} & \multirow[t]{2}{*}{ Dust } & \multicolumn{3}{|c|}{ Ionized emission } & \multirow{2}{*}{$\begin{array}{c}\text { X-ray } \\
\text { detection }^{(5)}\end{array}$} & \multirow{2}{*}{$\begin{array}{c}\phi_{\mathrm{EUV}}{ }^{(6)} \\
\left(10^{40}\right. \\
\left.\operatorname{erg~s}^{-1}\right)\end{array}$} \\
\hline & & & & $\begin{array}{c}\text { VLBA } \\
\text { detection } \\
\text { at } 5 \mathrm{GHz}^{(2)}\end{array}$ & Variability $^{(3)}$ & $\begin{array}{c}\text { Fully or } \\
\text { partially } \\
\text { non-thermal } \\
\text { emission }^{(4)}\end{array}$ & & \\
\hline \multicolumn{9}{|c|}{ Class 0} \\
\hline 1 & $\mathrm{~J} 162626.31-242430.7$ & $3.3 \pm 0.7 / 1.2 \pm 0.3$ & $\leq 21 \%$ & $\mathrm{~N}$ & $\mathrm{Y}$ & $\mathrm{Y}$ & & \\
\hline 2 & $\mathrm{~J} 162626.39-242430.8$ & $2.9 \pm 0.8 / 1.5 \pm 0.5$ & $\leq 21 \%$ & $\mathrm{~N}$ & & & & \\
\hline 3 & $\mathrm{~J} 162627.83-242359.3$ & $\geq 4.8 / \geq 1.7$ & $\leq 27 \%$ & & $\mathrm{Y}$ & $\mathrm{Y}$ & & \\
\hline \multicolumn{9}{|c|}{ Class I } \\
\hline 4 & J162617.24-242 346.0 & $\geq 2.9 / \geq 1.7$ & $\leq 28 \%$ & & & & & \\
\hline 5 & $\mathrm{~J} 162621.36-242304.7$ & $\geq 2.3 / \geq 1.0$ & $\leq 8 \%$ & & & & & \\
\hline 6 & $\mathrm{~J} 162621.72-242250.9$ & $0.6 \pm 0.5 / 0.5 \pm 0.2$ & $\leq 23 \%$ & $\mathrm{~N}$ & $\mathrm{Y}_{4.5 \mathrm{GHz}}{ }^{(\dagger)}$ & $\mathrm{Y}$ & & \\
\hline 7 & $162622.27-242407.1$ & & & & & & & \\
\hline 8 & J162623.58-242 439.9 & $>4.2 / 0.8 \pm 0.3$ & $\leq 0.2 \%$ & $\mathrm{~N}$ & $\mathrm{Y}$ & $\mathrm{Y}$ & $\mathrm{Y}$ & \\
\hline 9 & $162625.49-242301.6$ & $\ldots$ & $\leq 100 \%$ & & & & & \\
\hline 10 & J162625.63-242 429.4 & $1.2 \pm 0.7 / 0.3 \pm 0.2$ & $\leq 10 \%$ & Y & $\mathrm{Y}_{7.5 \mathrm{GHz}}{ }^{(\dagger)}$ & Y & & \\
\hline 11 & $162630.47-242257.1$ & $\ldots$ & $\leq 100 \%$ & & & & $\mathrm{Y}$ & \\
\hline \multicolumn{9}{|c|}{ Class II } \\
\hline 12 & $\mathrm{~J} 162610.32-242054.9$ & $2.3 \pm 0.6 / 1.4 \pm 0.2$ & $\leq 77 \%$ & $\mathrm{~N}$ & $Y^{(\dagger)}$ & $\mathrm{Y}$ & Y & $<74$ \\
\hline 13 & J162616.85-242 223.5 & $-5.7 \pm 1.0 /-1.9 \pm 0.4$ & $\leq 2 \%$ & $\mathrm{Y}$ & $\mathrm{Y}$ & $\mathrm{Y}$ & $\mathrm{Y}$ & \\
\hline 14 & 162617.06-242021.6 & $\ldots$ & $\ldots$ & & & & Y & $\lesssim 7$ \\
\hline 15 & $162618.82-242610.5$ & $\ldots$ & $\ldots$ & & & & & $\lesssim 7$ \\
\hline 16 & $162618.98-242414.3$ & $\ldots$ & $\leq 100 \%$ & & & & & $\lesssim 4$ \\
\hline 17 & $162621.53-242601.0$ & $\ldots$ & $\ldots$ & & & & Y & $\lesssim 7$ \\
\hline 18 & J162622.39-242 253.4 & $-5.5 \pm 0.4 /-2.4 \pm 0.2$ & $\leq 0.5 \%$ & $\mathrm{Y}$ & $\mathrm{Y}$ & $\mathrm{Y}$ & Y & $<71$ \\
\hline 19 & J162623.36-242 059.9 & $\geq 4.0 / \geq 1.4$ & $\leq 18 \%$ & & $\mathrm{Y}$ & $\mathrm{Y}$ & $\mathrm{Y}$ & $<45$ \\
\hline 20 & $\mathrm{~J} 162623.42-242101.9$ & $\geq 0.8 / \geq 0.3$ & $\leq 34 \%$ & & & & & $\lesssim 21$ \\
\hline 21 & $\mathrm{~J} 162624.04-242448.5$ & $\geq 1.9 / \geq 0.7$ & $\leq 1 \%$ & & & & $\mathrm{Y}$ & $\lesssim 28$ \\
\hline 22 & $\mathrm{~J} 162625.28-242445.4$ & $\ldots$ & $\ldots$ & & & & $\mathrm{Y}$ & $\lesssim 6$ \\
\hline 23 & 162627.81-242641.8 & $\ldots$ & $\ldots$ & & & & & $\lesssim 9$ \\
\hline 24 & $162637.79-242300.7$ & $\ldots$ & $\ldots$ & & & & & $\lesssim 10$ \\
\hline 25 & $162642.74-242427.7$ & $\ldots$ & $\ldots$ & & & & & $\lesssim 14$ \\
\hline 26 & $162642.89-242259.1$ & $\ldots$ & $\ldots$ & & & & & $\lesssim 17$ \\
\hline 27 & $162643.86-242450.7$ & $\ldots$ & $\ldots$ & & & & & $\lesssim 21$ \\
\hline \multicolumn{9}{|c|}{ Class III } \\
\hline 28 & $162615.81-241922.1$ & $\ldots$ & $\ldots$ & & & & $\mathrm{Y}$ & $\lesssim 15$ \\
\hline 29 & $162622.19-242352.4$ & $\ldots$ & $\ldots$ & & & & & $\lesssim 4$ \\
\hline 30 & $\mathrm{~J} 162625.23-242324.3$ & $\geq 0.3 / \geq 0.1$ & $\leq 2 \%$ & & & & $\mathrm{Y}$ & $\lesssim 20$ \\
\hline 31 & 162631.36-242530.2 & & & & & & $\mathrm{Y}$ & $\lesssim 6$ \\
\hline 32 & J162634.17-242 328.7 & $0.3 \pm 0.3 / 0.0 \pm 0.1$ & $\leq 0.02 \%$ & $\mathrm{Y}$ & $\mathrm{N}$ & $\mathrm{Y}$ & $\mathrm{Y}$ & \\
\hline
\end{tabular}

Notes. ${ }^{(1)}$ The first spectral index is calculated between 7.5 and $10 \mathrm{GHz}$, the second between 4.5 and $10 \mathrm{GHz}$. The uncertainties take into account both the fit uncertainty and the calibration uncertainty. The spectral indices can be significantly affected by variability, as the measurements were carried out at several epochs. ${ }^{(2)}$ Ortiz-León et al. (2017). Y for detection, $\mathrm{N}$ for non detection. ${ }^{(3)}$ Dzib et al. (2013) and this study. The sources with the symbol. ${ }^{(\dagger)}$ Correspond to the sources that only present variability $(>25 \%)$ in Dzib et al. $(2013) .{ }^{(4)} Y$ for the sources expected to present non-thermal emission based on the criteria discussed in the text (VLBA detection, variability, and spectral indices). Some of these sources could also be sources with thermal emission but abnormally high variability. ${ }^{(5)}$ Imanishi et al. (2003). ${ }^{(6)}$ EUV luminosities reaching the disks calculated from the fluxes or $3 \sigma$ noise levels measured in our VLA images at $10 \mathrm{GHz}(3 \mathrm{~cm})$ and using Eq. (2) of Pascucci et al. (2012) and a distance of $137 \mathrm{pc}$ for the Oph A cluster.

\subsection{Constraints on EUV disk photoevaporation}

The high sensitivity of our observations $\left(5 \mu \mathrm{Jy}\right.$ beam $^{-1}$ at the center of the field of view) and the proximity of this cloud $(137 \mathrm{pc})$ allow us to derive stringent constraints on the contribution of EUV photons on disk photoevaporation in the Oph A starforming region. As explained before, the radio emission of five of our detected Class II/III sources (J162610.32-242 054.9/\#12, J162616.85-242 223.5/\#13, J162622.39-242 253.4/\#18, J162623. $36-242059.9 / \# 19$, and J162634.17-242 328.7/\#32) is probably fully or partially non-thermal and we cannot exclude it for the three other detected sources. As such, the best constraints have come from the Class II/III objects we did not detect. 

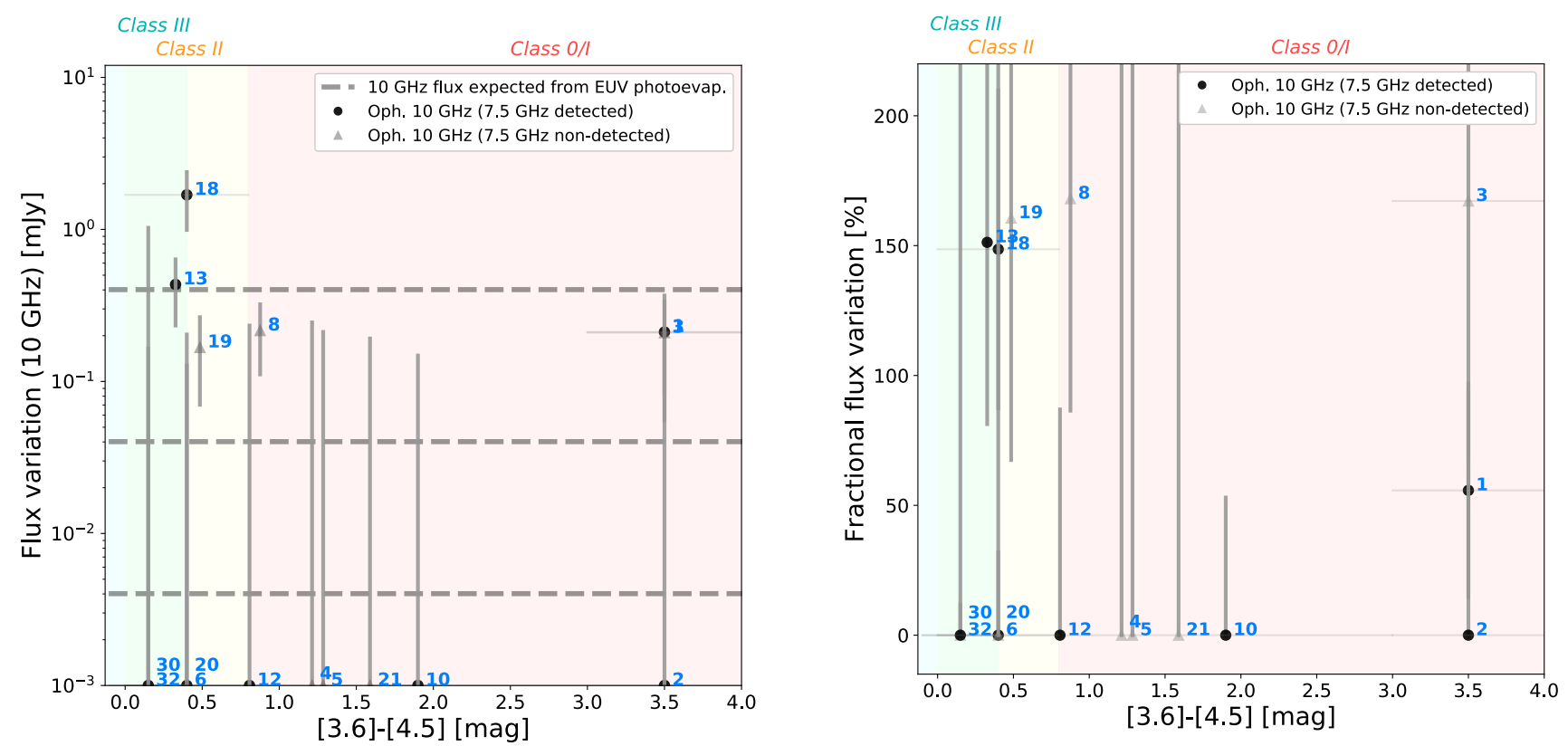

Fig. 4. Summary of observed radio flux variability (left panel) and fractional radio flux variability (right panel). We omitted sources which were not detected in both our $10 \mathrm{GHz}$ observations and the previous $7.5 \mathrm{GHz}$ observations of Dzib et al. (2013), since there is essentially no constraint on their time variability. For sources detected from at least one of those observations, we present the flux variation by calculating the average of the differences between the measured $10 \mathrm{GHz}$ flux in our VLA observations and the expected $10 \mathrm{GHz}$ flux derived by re-scaling the $7.5 \mathrm{GHz}$ flux from Dzib et al. (2013) to $10 \mathrm{GHz}$ assuming $\alpha=-0.1$ and 2.0. Vertical error bars take the measurement errors and the spectral index range [ -0.1 , 2.0] into consideration. Dashed lines in the left panel show the expected radio fluxes from EUV photoevaporation winds from protoplanetary disks, assuming the EUV flux $\Phi_{\mathrm{EUV}}=10^{40}$ (bottom), $10^{41}$ (middle), and $10^{42}$ photons s$^{-1}$ (top).

Following the approach of Pascucci et al. (2014) and GalvánMadrid et al. (2014), we estimate the expected radio continuum fluxes $F_{10 \mathrm{GHz}}$ for a particular EUV luminosity $\Phi_{\mathrm{EUV}}$ based on the following formulation:

$F_{10 \mathrm{GHz}}[\mu \mathrm{Jy}] \sim 4.0 \times 10^{-40}\left(\frac{137}{d[\mathrm{pc}]}\right)^{2}\left(\Phi_{\mathrm{EUV}}\left[\mathrm{s}^{-1}\right]\right)\left(\frac{10.0}{8.5}\right)^{\alpha}$,

where $d$ is the distance of the target source, and $\alpha$ is the spectral index of the free-free emission produced by the EUV photoevaporation. As an approximation, we tentatively consider $\alpha=0$, and note that our estimate of $F_{10 \mathrm{GHz}}$ is not especially sensitive to the exact value of $\alpha$ as long as $\alpha$ is in the range of [ -0.1 , 2.0]. We provide the estimates of $F_{10 \mathrm{GHz}}$ at $\Phi_{\mathrm{EUV}}=10^{40}, 10^{41}$, and $10^{42}$ photons s ${ }^{-1}$ for Figs. 3 and 4 . For Class II and III sources which were not detected in our observations, the respective $3 \sigma$ upper limits of $F_{10 \mathrm{GHz}}$ constrained their $\Phi_{\mathrm{EUV}}$ to be $\lesssim 4-21 \times 10^{40}$ photons $\mathrm{s}^{-1}$ (Fig. 3 and Table B.1). These upper limits are lower than those derived from previous observations toward $\mathrm{CrA}(<1-$ $4 \times 10^{41}$ photons s ${ }^{-1}$, Galván-Madrid et al. 2014). We note that typical EUV photoevaporation models require $\Phi_{\mathrm{EUV}}$ to be in the range of $10^{41}-10^{42} \mathrm{~s}^{-1}$ to disperse protoplanetary disks within a few Myrs (Font et al. 2004; Alexander et al. 2006; Alexander \& Armitage 2009). EUV-driven photoevaporation is, consequently, very unlikely to play a major role in the dispersal of these disks.

For the Class II and III sources that are detected in our $10 \mathrm{GHz}$ observations and do not necessarily exhibit nonthermal emission (J162623.42-242 102.0/\#20, J162624.04242 448.5/\#21, and J162625.23-242 324.3/\#30), if we assume that their $10 \mathrm{GHz}$ fluxes are dominated by photoevaporation winds, the corresponding $\Phi_{\mathrm{EUV}}$ values are well in the range required by the aforementioned models (Fig. 3). Hence, photoevaporation driven by EUV photons could be efficient enough to disperse these disks. Presently, however, we do not have sufficient constraints on the spectral indices of these detected sources to be able to tell what fractions of their radio fluxes come from constant EUV photoevaporation winds. Observationally, we also do not know yet whether the radio emission associated with EUV photoevaporating disks evolves over time.

\subsection{Constraints on $X$-ray disk photoevaporation}

Photoevaporation by X-ray photons is another process that may lead to the dispersal of protoplanetary disks. We listed in Table B.1 the observed X-ray luminosities found in the literature for the YSOs of Oph A (Imanishi et al. 2003). They range over $0.01-3 \times 10^{30} \mathrm{erg} \mathrm{s}^{-1}$. Pascucci et al. (2012) determined the relation between the incident X-ray photon luminosity $L_{\mathrm{X}}$ and the resulting free-free emission that a disk would emit:

$F_{10 \mathrm{GHz}}[\mu \mathrm{Jy}] \sim 3.3 \times 10^{-30}\left(\frac{137}{d[\mathrm{pc}]}\right)^{2}\left(L_{\mathrm{x}}\left[\mathrm{erg} \mathrm{s}^{-1}\right]\right)\left(\frac{10.0}{8.5}\right)^{\alpha}$.

Based on this equation and the level of non-detections in our Class II objects, the upper limits derived for the incident $\mathrm{X}$-ray photon luminosity are $\lesssim(7-25) \times 10^{30} \mathrm{erg} \mathrm{s}^{-1}$, i.e. about $1-2$ orders of magnitude higher than the observed values on average. Thus, we cannot exclude, with the present data, X-ray photoevaporation as a major mechanism in the dispersal of the disks. A series of more sensitive observations would be needed to determine its efficiency.

\subsection{Studying the photoevaporation of protoplanetary disks with the Square Kilometre Array}

In the future, the SKA will certainly revolutionize our understanding of the process of star and planet formation through radio 
emission studies. Here we discuss the potential of the SKA to investigate the photoevaporation of disks.

The free-free emission produced by a disk (at the distance of Oph A) with an X-ray luminosity of more than $10^{29} \mathrm{erg} \mathrm{s}^{-1}$ could be detected, for example, with an rms of $0.1 \mu \mathrm{Jy}$. Such a high level of sensitivity should be attainable in the future with the SKA. In particular, Hoare et al. (2015) estimates that a 1000-h deep field integration at the full resolution of SKA1-Mid ( $\sim 40$ mas, i.e. $\sim 5 \mathrm{AU}$ for the disks of Oph A) over a $2 \times 2.5 \mathrm{GHz}$ bandwidth from 8.8 to $13.8 \mathrm{GHz}$ would yield a noise level of $0.07 \mu \mathrm{Jy}$ beam $^{-1}$. Although the amount of time required appears significantly greater than the time dedicated to current radio projects, it should be noted that multiple projects will be carried out simultaneously with the SKA, and that a great number of sources will be covered in the same field with a single pointing. For example, the investigation of the photoevaporation in disk dispersal could be carried out simultaneously with high-priority studies of grain growth and the search for prebiotic molecules (Hoare et al. 2015).

With a single pointing, the SKA will cover a field of view of about 6 arcminutes (comparable to our four-pointing VLA mosaic). By targeting a rich region such as the Oph A cluster, a large number of disks (all the disks listed in this paper) can be observed simultaneously.

For bright radio emission sources, SKA will further provide good constraints on the instantaneous spectral indices over a wide range of frequency, useful data for gauging the fractional contributions of thermal and non-thermal emission mechanisms. An expansion of SKA1-Mid to $\sim 25 \mathrm{GHz}$ would provide even stronger constraints on the spectral indices resolved across the young stars, spatially separating the different components. Complementary observations will also be possible with the next generation VLA (ng-VLA, Murphy et al. 2018; Selina et al. 2018) above the highest SKA1-Mid band.

In addition, shallow (e.g., rms $\sim$ few $\mu \mathrm{Jy}$ ) but regularlyscheduled SKA monitoring surveys will provide, for the first time, statistics on how much time Class 0-III YSOs remain in the radio active or inactive states, and on the levels of dominant radio emission mechanisms and radio flux variability levels during these states.

Finally, the SKA1-Mid resolution will be around 40 mas, hence, making it possible to spatially separate the different contributions from flares, jet, wind and disk to some degree. Simultaneous observations of hydrogen radio recombination lines at the high-angular resolution of the SKA will also enable the separation of ionized gas emission from dust emission in disks, which will be key for these kinds of studies.

Obtaining photoevaporation rates should, consequently, be achievable with the power of the SKA, however separating out the role of each type (EUV/X-ray) may be more complicated. According to Pascucci et al. (2012), the EUV contribution should be a factor ten higher than the X-ray contribution. Photoevaporation models predict different mass-loss profiles, but the subtraction of the EUV contribution to the free-free emission (necessary to investigate the X-ray driven photoevaporation of disks) could turn out to be highly uncertain, since the EUV luminosity is unknown.

\section{Conclusions}

We carried out very sensitive continuum observations of the Oph A star-forming region at $10 \mathrm{GHz}$ with the VLA $\left(1 \sigma=5 \mu \mathrm{Jy}\right.$ beam $^{-1}$ at the center of the field of view). We detected sixteen YSOs and two extragalactic candidate sources.
Seven of the detected YSOs had not been detected in a previous VLA survey of this region at 4.5 and $7.5 \mathrm{GHz}$ by Dzib et al. (2013).

Using typical spectral indices for the possible components of radio emission, we constrained the origin of the emission detected at $10 \mathrm{GHz}$ to the YSOs. In general, dust emission contributes less than $30 \%$ of the total emission. The $10 \mathrm{GHz}$ emission appears to be mainly due to gyro-synchrotron emission from active magnetospheres, free-free emission from thermal jets or photoevaporative winds, or synchrotron emission due to accelerated cosmic-rays. Three of the YSOs show evidence of non-thermal emission. A comparison with the survey by Dzib et al. (2013) indicates that six of the sources show over $50 \%$ fractional radio flux variability, which is probably due to non-thermal emission.

The discussion surveys constraints on the EUV and X-ray photoevaporation mechanisms. For the Class II/III disks for which we detected no emission, the corresponding EUV luminosities are not sufficient to explain disk dispersal within a few Myrs through theoretical photoevaporation models. For the sources detected at $10 \mathrm{GHz}$ (with a potentially significant contribution of ionized thermal emission), the corresponding maximum $\Phi_{\mathrm{EUV}}$ values are within the range predicted by models. It is, however, currently unclear if EUV photoevaporating winds and their contributions to radio fluxes are constant over time. Even given the very high level of sensitivity in our observations, we have been unable to provide strong constraints on the efficiency of X-ray for disk dispersal. Observations of significantly greater sensitivity, which would also resolve the sources, are required to locate the different emission origins and constrain the efficiency of the photoevaporation mechanisms. With higher sensitivity and higher angular resolution, such future facilities as the SKA will make this a possibility.

Acknowledgements. This collaboration arose from discussions within the Cradle of Life Science Working Group of the SKA. The authors thank Hsieh Tien-Hao for providing the results of the classification method presented in Hsieh \& Lai (2013). The National Radio Astronomy Observatory is a facility of the National Science Foundation operated under cooperative agreement by Associated Universities. A.C. postdoctoral grant is funded by the ERC Starting Grant 3DICE (grant agreement 336474). I.J.-S. acknowledges the financial support received from the STFC through an Ernest Rutherford Fellowship (proposal number ST/L004801). L.L. acknowledges the financial support of DGAPA, UNAM (project IN112417), and CONACyT, México. A.C.T. acknowledges the financial support of the European Research Council (ERC; project PALs 320620). D.J. is supported by the National Research Council Canada and by an NSERC Discovery Grant. L.M.P. acknowledges support from CONICYT project Basal AFB-170002 and from FONDECYT Iniciación project \#11181068. A.P. acknowledges the support of the Russian Science Foundation project 18-12-00351. D.S. acknowledges support by the Deutsche Forschungsgemeinschaft through SPP 1833: Building a Habitable Earth (SE 1962/6-1). M.T. has been supported by the DISCSIM project, grant agreement 341137 funded by the European Research Council under ERC-2013-ADG. C.W. acknowledges support from the University of Leeds and the Science and Technology Facilities Council under grant number ST/R000549/1. This work was partly supported by the Italian Ministero dell'Istruzione, Università e Ricerca through the grant Progetti Premiali 2012 iALMA (CUP C52I13000140001), by the Deutsche Forschungs-gemeinschaft (DFG, German Research Foundation) - Ref no. FOR 2634/1 TE 1024/1-1, and by the DFG cluster of excellence Origin and Structure of the Universe (www.universe-cluster.de). This project has received funding from the European Union's Horizon 2020 research and innovation programme under the Marie Skłodowska-Curie grant agreement No 823823. This project has also been supported by the PRIN-INAF 2016 "The Cradle of Life - GENESIS-SKA (General Conditions in Early Planetary Systems for the rise of life with SKA)".

\section{References}

Alexander, R. D., \& Armitage, P. J. 2009, ApJ, 704, 989

Alexander, R. D., Clarke, C. J., \& Pringle, J. E. 2006, MNRAS, 369, 229

Alexander, R., Pascucci, I., Andrews, S., Armitage, P., \& Cieza, L. 2014,

Protostars and Planets VI (Tucson: University of Arizona Press), 475 
Allen, L. E., Calvet, N., D’Alessio, P., et al. 2004, ApJS, 154, 363

André, P., Montmerle, T., Feigelson, E. D., Stine, P. C., \& Klein, K.-L. 1988, ApJ, 335, 940

Anglada, G., Rodríguez, L. F., \& Carrasco-González, C. 2018, A\&ARv, 26, 3

Ansdell, M., Williams, J. P., Trapman, L., et al. 2018, ApJ, 859, 21

Carrasco-González, C., Rodríguez, L. F., Anglada, G., et al. 2010, Science, 330, 1209

Chiang, H.-F., Looney, L. W., \& Tobin, J. J. 2012, ApJ, 756, 168

Choi, M., Tatematsu, K., Hamaguchi, K., \& Lee, J.-E. 2009, ApJ, 690, 1901

Cox, E. G., Harris, R. J., Looney, L. W., et al. 2017, ApJ, 851, 83

Dzib, S. A., Loinard, L., Mioduszewski, A. J., et al. 2013, ApJ, 775, 63

Ercolano, B., Weber, M. L., \& Owen, J. E. 2018, MNRAS, 473, L64

Evans, II, N. J., Dunham, M. M., Jørgensen, J. K., et al. 2009, ApJS, 181, 321

Font, A. S., McCarthy, I. G., Johnstone, D., \& Ballantyne, D. R. 2004, ApJ, 607, 890

Forbrich, J., Massi, M., Ros, E., Brunthaler, A., \& Menten, K. M. 2007, A\&A, 469,985

Forbrich, J., Reid, M. J., Menten, K. M., et al. 2017, ApJ, 844, 109

Friesen, R. K., Di Francesco, J., Bourke, T. L., et al. 2014, ApJ, 797, 27

Friesen, R. K., Pon, A., Bourke, T. L., et al. 2018, ApJ, 869, 158

Gagné, M., Skinner, S. L., \& Daniel, K. J. 2004, ApJ, 613, 393

Galván-Madrid, R., Liu, H. B., Manara, C. F., et al. 2014, A\&A, 570, L9

Galván-Madrid, R., Liu, H. B., Izquierdo, A. F., et al. 2018, ApJ, 868, 39

Gibb, A. G. 1999, MNRAS, 304, 1

Güdel, M. 2002, ARA\&A, 40, 217

Guilloteau, S., Dutrey, A., Piétu, V., \& Boehler, Y. 2011, A\&A, 529, A105

Gutermuth, R. A., Megeath, S. T., Myers, P. C., et al. 2009, ApJS, 184, 18

Haisch, Jr. K. E., Jayawardhana, R., \& Alves, J. 2005, ApJ, 627, L57

Hoare, M., Perez, L., Bourke, T. L., et al. 2015, Advancing Astrophysics with the Square Kilometre Array (AASKA14), 115

Hollenbach, D., Johnstone, D., Lizano, S., \& Shu, F. 1994, ApJ, 428, 654

Hsieh, T.-H., \& Lai, S.-P. 2013, ApJS, 205, 5

Imanishi, K., Nakajima, H., Tsujimoto, M., Koyama, K., \& Tsuboi, Y. 2003 PASJ, 55, 653

Johansen, A., Blum, J., Tanaka, H., et al. 2014, Protostars and Planets VI (Tucson: University of Arizona Press), 547

Jørgensen, J. K., Johnstone, D., Kirk, H., et al. 2008, ApJ, 683, 822

Kirk, H., Dunham, M. M., Di Francesco, J., et al. 2017, ApJ, 838, 114

Kirk, H., Hatchell, J., Johnstone, D., et al. 2018, ApJS, 238, 8

Kruger, A. J., Richter, M. J., Seifahrt, A., et al. 2012, ApJ, 760, 88

Leous, J. A., Feigelson, E. D., Andre, P., \& Montmerle, T. 1991, ApJ, 379, 683

Li, J. I., Liu, H. B., Hasegawa, Y., \& Hirano, N. 2017, ApJ, 840, 72

Liu, H. B., Galván-Madrid, R., Forbrich, J., et al. 2014, ApJ, 780, 155

Loinard, L., Torres, R. M., Mioduszewski, A. J., \& Rodríguez, L. F. 2008, ApJ, 675, L29

Macías, E., Anglada, G., Osorio, M., et al. 2016, ApJ, 829, 1

McMullin, J. P., Waters, B., Schiebel, D., Young, W., \& Golap, K. 2007, in Astronomical Data Analysis Software and Systems XVI, eds. R. A. Shaw, F. Hill, \& D. J. Bell, ASP Conf. Ser., 376, 127

Miotello, A., Testi, L., Lodato, G., et al. 2014, A\&A, 567, A32

Motte, F., Andre, P., \& Neri, R. 1998, A\&A, 336, 150

Murphy, E. J., Bolatto, A., Chatterjee, S., et al. 2018, in Science with a Next Generation Very Large Array, ed. E. Murphy, ASP Conf. Ser., 517, 3

Ortiz-León, G. N., Loinard, L., Kounkel, M. A., et al. 2017, ApJ, 834, 141

Owen, J. E., Ercolano, B., \& Clarke, C. J. 2011, MNRAS, 412, 13

Owen, J. E., Clarke, C. J., \& Ercolano, B. 2012, MNRAS, 422, 1880

Padovani, M., \& Galli, D. 2018, A\&A, 620, L4

Padovani, M., Marcowith, A., Hennebelle, P., \& Ferrière, K. 2016, A\&A, 590, A8

Pascucci, I., Gorti, U., \& Hollenbach, D. 2012, ApJ, 751, L42

Pascucci, I., Ricci, L., Gorti, U., et al. 2014, ApJ, 795, 1

Pascucci, I., Testi, L., Herczeg, G. J., et al. 2016, ApJ, 831, 125

Pattle, K., Ward-Thompson, D., Kirk, J. M., et al. 2015, MNRAS, 450, 1094

Pérez, L. M., Carpenter, J. M., Chandler, C. J., et al. 2012, ApJ, 760, L17

Ribas, Á., Bouy, H., \& Merín, B. 2015, A\&A, 576, A52

Ricci, L., Testi, L., Natta, A., \& Brooks, K. J. 2010, A\&A, 521, A66

Russell, S. S., Hartmann, L., Cuzzi, J., et al. 2006, Timescales of the Solar Protoplanetary Disk, eds. D. S. Lauretta, \& H. Y. McSween (Tucson: University of Arizona Press), 233

Selina, R. J., Murphy, E. J., McKinnon, M., et al. 2018, in Science with a Next Generation Very Large Array, ed. E. Murphy, ASP Conf. Ser., 517, 15

Strom, K. M., Strom, S. E., Edwards, S., Cabrit, S., \& Skrutskie, M. F. 1989, AJ, 97, 1451

Tazzari, M., Testi, L., Ercolano, B., et al. 2016, A\&A, 588, A53

Testi, L., Birnstiel, T., Ricci, L., et al. 2014, Protostars and Planets VI (Tucson: University of Arizona Press), 339

Tobin, J. J., Dunham, M. M., Looney, L. W., et al. 2015, ApJ, 798, 61
Tobin, J. J., Chandler, C. J., Wilner, D. J., et al. 2013, ApJ, 779, 93

Tychoniec, Ł., Tobin, J. J., Karska, A., et al. 2018, ApJS, 238, 19

van der Marel, N., Williams, J. P., Ansdell, M., et al. 2018, ApJ, 854, 177

Wilking, B. A., Gagné, M., \& Allen, L. E. 2008, Star Formation in the $\rho$ Ophiuchi

Molecular Cloud (California: Astronomical Society of the Pacific), 351

Williams, J. P., \& Cieza, L. A. 2011, ARA\&A, 49, 67

1 Laboratoire d'Astrophysique de Bordeaux, Univ. Bordeaux, CNRS, B18N, allée Geoffroy Saint-Hilaire, 33615 Pessac, France

e-mail: audrey . coutens@u-bordeaux . fr

2 European Southern Observatory (ESO), Karl-Schwarzschild-Str. 2, 85748 Garching, Germany

3 School of Physics and Astronomy, Queen Mary University of London, Mile End Road, London E1 4NS, UK

4 SKA Organisation, Jodrell Bank Observatory, Lower Withington, Macclesfield, Cheshire SK11 9DL, UK

5 Centre for Astrophysics Research, University of Hertfordshire, College Lane, Hatfield AL10 9AB, UK

6 School of Physics and Astronomy, University of Leeds, Leeds LS2 9JT, UK

7 Instituto de Radioastronomía y Astrofísica, Universidad Nacional Autónoma de México, Morelia 58089, México

8 Instituto de Astronomía, Universidad Nacional Autónoma de Mexico, Apartado Postal 70-264, Ciudad de México 04510, México

9 INAF - Osservatorio Astrofisico di Arcetri, Largo Enrico Fermi 5, 50125 Florence, Italy

10 Department of Astronomy, University of Geneva, Ch. des Maillettes 51, 1290 Versoix, Switzerland

11 Department of Astronomy, University of Geneva, Ch. d'Ecogia 16, 1290 Versoix, Switzerland

12 Max-Planck-Institüt für extraterrestrische Physik, Giessenbachstrasse 1, 85748 Garching, Germany

13 Observatorio Astronómico Nacional (OAG-IGN), Alfonso XII 3, 28014 Madrid, Spain

14 Université Grenoble Alpes, Institut de Planétologie et d'Astrophysique de Grenoble (IPAG), 38401 Grenoble, France

15 NRC Herzberg Astronomy and Astrophysics, 5071 West Saanich Rd, Victoria, BC, V9E 2E7, Canada

16 Department of Physics and Astronomy, University of Victoria, Victoria, BC, V8P 5C2, Canada

17 Leiden Observatory, Leiden University, PO Box 9513, 2300 RA Leiden, The Netherlands

18 Anton Pannekoek Institute for Astronomy, University of Amsterdam, Science Park 904, 1098 XH Amsterdam, The Netherlands

19 Lund Observatory, Lund University, Box 43, 22100 Lund, Sweden

${ }^{20}$ Centre for Astrophysics and Supercomputing, Swinburne University of Technology, Hawthorn, Victoria 3122, Australia

21 Departamento de Astronomía, Universidad de Chile, Camino El Observatorio 1515, Las Condes, Santiago, Chile

22 Ural Federal University, 620002, 19 Mira street, Yekaterinburg, Russia

23 Department of Physics and Astronomy, University College London, Gower St., London WC1E 6BT, UK

24 Department of Chemistry, Ludwig Maximilian University, Butenandtstr. 5-13, 81377 München, Germany

25 Max Planck Institute for Astronomy, Königstuhl 17, 69117, Heidelberg, Germany

26 Institute of Astronomy, University of Cambridge, Madingley Road, CB3 0HA, Cambridge, UK

27 NRAO, 520 Edgemont Road Charlottesville, VA 22903-2475 USA

28 ASTRON Netherlands Institute for Radio Astronomy, Oude Hoogeveensedijk 4, 7991 PD Dwingeloo, The Netherlands

29 Joint Institute for VLBI ERIC (JIVE), Oude Hoogeveensedijk 4, 7991 PD Dwingeloo, The Netherlands

30 Department of Space, Earth and Environment, Chalmers University of Technology, Onsala Space Observatory, 43992 Onsala, Sweden

31 Harvard-Smithsonian Center for Astrophysics, 60 Garden Street, Cambridge, MA 02 138, USA 
A. Coutens et al.: VLA cm-wave survey of young stellar objects in the Oph A cluster

\section{Appendix A: Image component sizes obtained}

with imfit

Table A.1. Image component sizes (deconvolved from beam) obtained with imfit.

\begin{tabular}{ccccc}
\hline \hline$\#$ & Source & $\begin{array}{c}\text { Major axis } \\
F W H M\left({ }^{\prime \prime}\right)\end{array}$ & $\begin{array}{c}\text { Minor axis } \\
F W H M\left({ }^{\prime \prime}\right)\end{array}$ & $\begin{array}{c}\text { Position } \\
\text { angle }\left(^{\circ}\right)\end{array}$ \\
\hline 1 & $\mathrm{~J} 162626.31-242430.7$ & $0.78 \pm 0.09$ & $0.52 \pm 0.09$ & $76 \pm 15$ \\
2 & $\mathrm{~J} 162626.39-242430.8$ & $0.83 \pm 0.14$ & $0.45 \pm 0.11$ & $67 \pm 16$ \\
\hline 6 & $\mathrm{~J} 162621.72-242250.9$ & $0.97 \pm 0.11$ & $0.57 \pm 0.05$ & $37 \pm 8$ \\
8 & $\mathrm{~J} 162623.58-242439.9$ & $0.94 \pm 0.20$ & $0.75 \pm 0.14$ & $36 \pm 77$ \\
10 & $\mathrm{~J} 162625.63-242429.4$ & $1.06 \pm 0.22$ & $0.69 \pm 0.23$ & $85 \pm 28$ \\
\hline 18 & $\mathrm{~J} 162622.39-242253.4$ & $1.08 \pm 0.14$ & $0.52 \pm 0.05$ & $26 \pm 6$ \\
21 & $\mathrm{~J} 162624.04-242448.5$ & $1.37 \pm 0.40$ & $0.47 \pm 0.15$ & $52 \pm 12$ \\
\hline 32 & $\mathrm{~J} 162634.17-242328.7$ & $0.65 \pm 0.02$ & $0.09 \pm 0.01$ & $25.6 \pm 0.5$ \\
\hline 42 & $\mathrm{~J} 162635.33-242405.3$ & $0.55 \pm 0.14$ & $0.25 \pm 0.07$ & $50 \pm 16$ \\
\hline
\end{tabular}

Notes. Sources are listed in the same order as in Table B.1. 


\section{Appendix B: Additional table}

Table B.1. Catalog of sources observed in field of view of our observations grouped in categories.

\begin{tabular}{|c|c|c|c|c|c|c|c|c|c|c|}
\hline$\#$ & $\begin{array}{l}\text { Source }{ }^{(0)} \\
(\mathrm{J} 2000 \text { coordinates) }\end{array}$ & $\begin{array}{c}\text { Flux }(\mathrm{mJy})^{(1)} \\
10.0 \mathrm{GHz}\end{array}$ & $\begin{array}{c}\text { Flux }(\mathrm{mJy})^{(1)} \\
7.5 \mathrm{GHz} \\
\end{array}$ & $\begin{array}{c}\text { Flux }(\mathrm{mJy})^{(1)} \\
\quad 4.5 \mathrm{GHz} \\
\end{array}$ & $\begin{array}{l}\text { Flux }(\mathrm{mJy})^{(1)} \\
107 \mathrm{GHz}\end{array}$ & $\begin{array}{l}\text { Source } \\
\text { type }^{(2)}\end{array}$ & Refs. ${ }^{(3)}$ & Variable $^{(4)}$ & $\begin{array}{c}L_{\mathrm{X}}{ }^{(5)} \\
\left(10^{29} \mathrm{erg} \mathrm{s}^{-1}\right) \\
\end{array}$ & Other names \\
\hline 1 & J162626.31-242 430.7 & $0.485 \pm 0.033$ & $0.189 \pm 0.034$ & $0.189 \pm 0.034$ & $59.82 \pm 0.47^{(1)}$ & YSO $0 ?^{(8)}$ & $\mathrm{a}$ & $\mathrm{Y}$ & & VLA1623 B \\
\hline 2 & J162626.39-242 430.8 & $0.289 \pm 0.030$ & $0.125 \pm 0.025$ & $0.087 \pm 0.030$ & $59.82 \pm 0.47^{(1)}$ & YSO 0 & a & $\mathrm{U}$ & & VLA1623 A \\
\hline 3 & $\mathrm{~J} 162627.83-242359.4$ & $0.230^{(7)}$ & $\lessgtr 0.051$ & $\lesssim 0.051$ & $23.13 \pm 0.46$ & $\mathrm{YSO} / \mathrm{PC}^{(9)}$ & $\mathrm{b}$ & & & SM1 \\
\hline 4 & J162617.23-242 345.7 & $0.140^{(7)}$ & $\lesssim 0.051$ & $\lesssim 0.051$ & $14.46 \pm 0.29$ & YSO I & $\mathrm{c}, \mathrm{d}$ & & & $\begin{array}{l}\text { A-MM33, CRBR12, } \\
\text { ISO-Oph } 21\end{array}$ \\
\hline 5 & J162621.36-242 304.7 & $0.120^{(7)}$ & $\lesssim 0.051$ & $\lesssim 0.051$ & $3.48 \pm 0.67$ & YSO I/0-I & $\mathrm{c} / \mathrm{d}$ & & & GSS 30-IRS1, Elias 21 \\
\hline 6 & J162621.72-242 250.9 & $0.364 \pm 0.030$ & $0.304 \pm 0.029$ & $0.238 \pm 0.017$ & $30.71 \pm 0.63$ & YSO I/FS & $a / c$ & $\mathrm{Y}_{4.5 \mathrm{GHz}}{ }^{(10)}$ & & GSS 30-IRS3, LFAM1 \\
\hline 7 & $162622.27-242407.1$ & $\lesssim 0.015$ & $\lesssim 0.051$ & $\lesssim 0.051$ & $\lesssim 0.16$ & YSO I-FS & $\mathrm{c}$ & & & CRBR25 \\
\hline 8 & J162623.58-242 439.9 & $0.237 \pm 0.035$ & $<0.06$ & $0.125 \pm 0.015$ & $\lesssim 0.16$ & YSO 0-I/FS & $\mathrm{d} / \mathrm{a}, \mathrm{c}$ & $\mathrm{Y}$ & 10.8 & LFAM 3 \\
\hline 9 & 162625.49-242 301.6 & $\lesssim 0.015$ & $\lesssim 0.051$ & $\lesssim 0.051$ & $9.17 \pm 0.44$ & YSO I & $\mathrm{c}$ & & & CRBR36 \\
\hline 10 & J162625.63-242 429.4 & $0.277 \pm 0.041$ & $0.198 \pm 0.023$ & $0.218 \pm 0.014$ & $11.24 \pm 0.44$ & YSO I & $\mathrm{a}, \mathrm{d}$ & $\mathrm{Y}_{7.5 \mathrm{GHz}}{ }^{(10)}$ & & VLA1623 W, LFAM 4 \\
\hline 11 & $162630.47-242257.1$ & $\lesssim 0.021$ & $\lessgtr 0.051$ & $\lesssim 0.051$ & $11.22 \pm 0.44$ & YSO FS/0-I & $\mathrm{c} / \mathrm{d}$ & & 4.8 & $\ldots$ \\
\hline 12 & J162610.32-242054.9 & $0.307^{(7)}$ & $0.160 \pm 0.022$ & $0.100 \pm 0.012$ & $27.25 \pm 0.35$ & YSO II & $\mathrm{a}, \mathrm{c}, \mathrm{d}$ & $\mathrm{Y}$ & 9.5 & GSS 26 \\
\hline 13 & $\mathrm{~J} 162616.85-242223.5$ & $0.070^{(7)}$ & $0.360 \pm 0.024$ & $0.337 \pm 0.017$ & $\lesssim 0.16$ & YSO II & $\mathrm{a}, \mathrm{c}, \mathrm{d}$ & $\mathrm{Y}$ & 16.3 & GSS 29, LFAM p1 \\
\hline 14 & 162617.06-242 021.6 & $\lessgtr 0.030$ & $\lesssim 0.051$ & $\lesssim 0.051$ & $\lesssim 0.16$ & YSO II & $\mathrm{c}$ & & 10.7 & DoAr 24 \\
\hline 15 & $162618.82-242610.5$ & $\lesssim 0.030$ & $\lesssim 0.051$ & $\lesssim 0.051$ & $\lesssim 0.16$ & YSO II & $\mathrm{c}$ & & & $\ldots$ \\
\hline 16 & 162618.98-242 414.3 & $\lesssim 0.015$ & $\lesssim 0.051$ & $\$ 0.051$ & $5.96 \pm 0.98$ & YSO II-FS & c & & & CRBR15 \\
\hline 17 & 162621.53-242 601.0 & $\lesssim 0.030$ & $\lesssim 0.051$ & $\lesssim 0.051$ & $\ldots$ & YSO II & c & & $0.1-0.6$ & \\
\hline 18 & J162622.39-242 253.4 & $0.292 \pm 0.027$ & $1.42 \pm 0.07$ & $2.02 \pm 0.10$ & $\lesssim 0.16$ & YSO II & $\mathrm{a}, \mathrm{c}$ & $\mathrm{Y}$ & 51.5 & $\begin{array}{l}\text { GSS 30-IRS2, VSSG12, } \\
\text { ISO-Oph } 34 \text {, LFAM } 2\end{array}$ \\
\hline 19 & $\mathrm{~J} 162623.36-242059.9^{(6)}$ & $0.188^{(7)}$ & $\lesssim 0.051$ & $\lesssim 0.051$ & $\ldots$ & YSO II & $\mathrm{c}, \mathrm{d}$ & & 4.7 & GSS 31a, DoAr 24Ea \\
\hline 20 & J162623.42-242 102.0 & $0.085^{(7)}$ & $\lesssim 0.051$ & $\lesssim 0.051$ & $\ldots$ & YSO II? & e & & & GSS 31b, DoAr 24Eb \\
\hline 21 & J162624.04-242 448.5 & $0.115 \pm 0.027$ & $\lessgtr 0.051$ & $\lessgtr 0.051$ & $\lesssim 0.16$ & YSO II/FS & $\mathrm{c} / \mathrm{d}$ & & 29.2 & S2 \\
\hline 22 & $\mathrm{~J} 162625.28-242445.4^{(11)}$ & $\lesssim 0.024$ & $\lesssim 0.051$ & $\lesssim 0.051$ & $\lesssim 0.16$ & YSO II & $\mathrm{c}$ & & 0.5 & $\ldots$ \\
\hline 23 & $162627.81-242641.8$ & $\lesssim 0.036$ & $\lesssim 0.051$ & $\lesssim 0.051$ & $\ldots$ & YSO II & $\mathrm{c}$ & & & $\ldots$ \\
\hline 24 & $162637.79-242300.7$ & $\lesssim 0.042$ & $\lesssim 0.051$ & $\lesssim 0.051$ & $\lessgtr 0.16$ & YSO II & c & & & LFAM p2 \\
\hline 25 & $162642.74-242427.7$ & $\lesssim 0.060$ & $\lesssim 0.051$ & $\lesssim 0.051$ & $\lesssim 0.16$ & YSO II & c & & & $\ldots$ \\
\hline 26 & $162642.89-242259.1$ & $\lesssim 0.069$ & $\lesssim 0.051$ & $\lesssim 0.051$ & $\lesssim 0.16$ & YSO II & $\mathrm{c}$ & & & $\ldots$ \\
\hline 27 & 162643.86-242 450.7 & $\lesssim 0.084$ & $\lesssim 0.051$ & $\lesssim 0.051$ & $\lesssim 0.16$ & YSO II & $\mathrm{c}$ & & & $\ldots$ \\
\hline 28 & 162615.81-241 922.1 & $\lesssim 0.063$ & $\lesssim 0.051$ & $\lesssim 0.051$ & $\lesssim 0.16$ & YSO III & $\mathrm{c}$ & & 3.1 & $\ldots$ \\
\hline 29 & $162622.19-242352.4$ & $\lessgtr 0.015$ & $\lesssim 0.051$ & $\lesssim 0.051$ & $\lesssim 0.16$ & YSO III & c & & & $\ldots$ \\
\hline 30 & J162625.23-242 324.3 & $0.081^{(7)}$ & $\lesssim 0.051$ & $\lesssim 0.051$ & $\lesssim 0.16$ & YSO III/FS & $\mathrm{c} / \mathrm{d}$ & & 6.0 & $\ldots$ \\
\hline 31 & $162631.36-242530.2$ & $\lessgtr 0.024$ & $\lesssim 0.051$ & $\lesssim 0.051$ & $\lesssim 0.16$ & YSO III & $\mathrm{c}$ & & 0.2 & $\ldots$ \\
\hline 32 & J162634.17-242 328.7 & $7.75 \pm 0.11$ & $7.07 \pm 0.35$ & $7.98 \pm 0.40$ & $\$ 0.16$ & YSO III & $\mathrm{a}, \mathrm{c}$ & $\mathrm{N}$ & 22.6 & S1 \\
\hline 33 & $162614.63-242507.5$ & $\$ 0.024$ & $\$ 0.051$ & $\lesssim 0.051$ & $\lesssim 0.16$ & YSO U & $\mathrm{f}$ & & & $\ldots$ \\
\hline 34 & $162625.99-242340.5$ & $\lesssim 0.015$ & $\lesssim 0.051$ & $\lesssim 0.051$ & $\lesssim 0.16$ & YSO U & $\mathrm{g}$ & & & $\ldots$ \\
\hline 35 & $162632.53-242635.4$ & $\lesssim 0.045$ & $\lesssim 0.051$ & $\lesssim 0.051$ & $\lesssim 0.16$ & YSO U & $\mathrm{c}$ & & & CRBR40 \\
\hline 36 & $162638.80-242322.7$ & $\lesssim 0.045$ & $\lesssim 0.051$ & $\lesssim 0.051$ & $\ldots$ & YSO U & $\mathrm{c}$ & & & $\ldots$ \\
\hline 37 & $162639.92-242233.4$ & $\lesssim 0.078$ & $\lesssim 0.051$ & $\lesssim 0.051$ & $\ldots$ & YSO U & c & & & $\ldots$ \\
\hline 38 & $162608.04-242523.1$ & $\lesssim 0.051$ & $\leq 0.05$ & $0.103 \pm 0.014$ & & EG? & a & $\mathrm{Y}$ & & \\
\hline 39 & $\mathrm{~J} 162629.62-242317.3$ & $0.091^{(4)}$ & $0.124 \pm 0.018$ & $0.228 \pm 0.014$ & & EG? & $\mathrm{a}$ & $\mathrm{Y}$ & & \\
\hline 40 & J162630.59-242 023.0 & $\lesssim 0.045$ & $0.064 \pm 0.017$ & $0.098 \pm 0.013$ & & EG? & $\mathrm{a}$ & $\mathrm{Y}$ & & \\
\hline 41 & J162634.95-242 655.3 & $\lesssim 0.069$ & $0.100 \pm 0.013$ & $0.197 \pm 0.019$ & & EG? & a & $\mathrm{Y}$ & & \\
\hline 42 & J162635.33-242 405.3 & $0.377 \pm 0.039$ & $0.329 \pm 0.033$ & $0.650 \pm 0.038$ & & EG? & a & $\mathrm{Y}$ & & \\
\hline
\end{tabular}

Notes. ${ }^{(0)}$ The source name starts with J when detected with the VLA, either in this study or in Dzib et al. (2013). The rest of the name correspond to the J2000 RA-Dec coordinates hhmmss.ss-ddmmss.s. When undetected with VLA, we used the coordinates given in the references listed in Col. 8. ${ }^{(1)}$ The fluxes measured at $10.0 \mathrm{GHz}$ were derived with Gaussian fit. The uncertainties correspond to the fit uncertainties only. The fluxes measured at 4.5 and $7.5 \mathrm{GHz}$ come from Dzib et al. (2013), while the ones at $107 \mathrm{GHz}$ come from Kirk et al. (2017). It should be noted that the flux measured at $107 \mathrm{GHz}$ for J162626.31-242 430.7 and J162626.39-242 430.8 includes the two sources. The upper limits correspond to the $3 \sigma$ levels $\left(1 \sigma=17 \mu \mathrm{Jy}_{\text {beam }}{ }^{-1}\right.$ for both frequencies) measured in the three-epoch combined images from Dzib et al. $(2013)$. ${ }^{(2)}$ The source is either YSO (Young Stellar Object) or EG (Extragalactic candidate). The YSOs are classified into: PC (prestellar core), 0 (Class 0 protostar), I (Class I protostar), FS (Flat Spectrum), II (Class II protostar), III (Class III protostar), U (Unknown classification for the YSO candidates). ${ }^{(4)}$ Variability taken from Dzib et al. (2013). A source is considered variable when its variability fraction is $\geq 25 \%$. Legend: $\mathrm{Y}-$ variable; $\mathrm{N}-$ not variable; $\mathrm{U}$ - unknown. ${ }^{(5)} \mathrm{X}$-ray luminosity in the $0.5-9.0 \mathrm{keV}$ (Imanishi et al. 2003). ${ }^{(6)}$ This source could be a binary. The flux given here corresponds to the total flux. ${ }^{(7)}$ Contrary to the other sources, the fluxes of these objects were integrated over a circular area selected with CASA due to their particular structure, which may be caused by residual phase errors, or due to a very uncertain Gaussian fit. ${ }^{(8)}$ This source may be a young star or an outflow knot feature. ${ }^{(9)}$ This source was proposed to contain an extremely young, deeply embedded protostellar object (Friesen et al. 2014).

${ }^{(10)}$ Source variable only at the indicated frequency. ${ }^{(11)}$ Source only detected in epoch 3 with a flux of 0.4 Jy.

References. ${ }^{(3)}$ References for the YSO classification : (a) Dzib et al. (2013), (b) Friesen et al. (2014), (c) Wilking et al. (2008), (d) Hsieh \& Lai (2013), (e) Kruger et al. (2012), (f) Jørgensen et al. (2008), (g) Evans et al. (2009). 